\title{
RECUPERAÇÃO ESPONTANEA DA FUNÇÃO NAS LESÕES DO SISTEMA NERVOSO CENTRAL
}

\author{
Abrão AnghinaH *
}

Os mecanismos básicos do retorno espontâneo da função após lesões do sistema nervoso foram, em parte, comprovados pelos neurofisiologistas em animais de laboratório. Estes achados, entretanto, não puderam ser até hoje correlacionados com os dados fornecidos pela observação clínica diária, pois numerosos são os casos nos quais a recuperação espontânea da função ocorre em circunstâncias inexplicáveis. Dois grupos de hipóteses procuram explicar estes mecanismos: as que admitem a reorganização espontânea do tecido nervoso residual e integro com desenvolvimento ulterior de novas conexões; as que se baseiam na substituição funcional - função vicariante - isto é, admitem que a função perdida em decorrência da lesão de um dado centro poderá retornar desde que seja substituída por outro centro integro localizado em qualquer ponto do sistema nervoso central.

\section{REORGANIZAÇAO ESPONTANEA}

Em 1910, Von Monakow (cit. por Lashley ${ }^{20}$ ) procurou esclarecer a recuperação da função em pacientes com traumatismo raquimedular nos quais havia decorrido tempo regularmente longo após a "fase de choque"; admitia Von Monakow que, durante o choque medular, haveria bloqueio no trânsito de impulsos facilitadores para um dado centro nervoso e, consequentemente, depressão de suas funçōes; durante a evolução ocorria, gradativamente, a dissolução (diásquise) do "estado de depressão", dando lugar à recuperação das funções. Reise (cit. por Haymaker ${ }^{14}$ ) interpretou de forma diversa o significado da diásquise; na dissolução ocorria a liberação de centros que eram inibidos por aqueles que tinham sido lesados.

Kennard (1938) 17 investigou a reorganização da função motora no córtex cerebral de macacos. Após a ablação das áreas 4 e 6 de Brodmann de macacos recém-nascidos, Kennard verificou que os animais conservavam a capacidade de executar movimentos coordenados e que os mesmos se desenvolviam com o decorrer do tempo, atribuindo esse fato à reorganização de outras áreas corticais pela dificuldade de executar movimentos mais delica-

Clínica Neurológica (Prof. Horácio M. Canelas) do Departamento de Neuropsiquiatria da Faculdade de Medicina da Universidade de São Paulo: *Docente-Livre. 
dos, em particular a preensão; verificou ainda que a ablação total das áreas motoras no macaco adulto promovia, decorrido algum tempo, o aparecimento de um tipo de motricidade em tudo semelhante à do recém-nascido, isto é, retorno da "preensão forçada", bem como tendência para agarrar e elevar o corpo; tais movimentos persistiam indefinidamente apesar da presença de movimentos voluntários intencionais. Estes trabalhos fundamentais permitiram analisar e distinguir as semelhanças e diferenças funcionais existentes entre córtex cerebral do recém-nascido e do adulto. Faltavam, entretanto, as bases anatômicas para apoiar esta teoria.

Em 1958, Mc Couch \& col. ${ }^{24}$, em investigações nas quais promoviam a secção parcial da medula espinhal de macacos e gatos, verificaram que, decorridas 3 semanas (exceção das lesões na coluna dorsal), a aplicação de uma única descarga na raiz dorsal promovia aumento da amplitude do potencial pré-sináptico que se associava à atividade normal das terminações aferentes; por outro lado, o aumento do potencial internuncial não excedia aquele do terminal aferente e, às vezes, sua intensidade estava diminuida. Os exames histológicos da medula espinal destes animais revelaram aumento na distribuição de aferentes no lado correspondente à hemisecção. O brotamento do terminal aferente para preencher os claros deixados pela degeneraçāo dos tratos descendentes seria a principal causa da espasticidade na paraplegia. O mesmo fato foi observado em fibras normais, após degeneração de axônios recém-nascidos. Lesões confinadas à coluna dorsal também resultam em brotamento se ao mesmo tempo ocorrer degeneração de tratos descendentes; nesta última eventualidade, mesmo que haja degeneração de tratos descendentes, o brotamnto não desenvolve conexões funcionais. Diante destas observaçōes, Mac Couch \& col. ${ }^{24}$ passaram a admitir que a hiperatividade dos reflexos miotáticos que evoluem para a espasticidade se deve ao fato de a superfície dos pericários espinais, bem como as porçōes proximais de seus dendritos, serem invadidas por placas terminais até o ponto de saturação e, além disso, a substituição das fibras degeneradas dos tratos descendentes por brotamento de fibras recém-nascidas da raiz posterior que, por sua vez, podem atingir acentuado grau de controle dos arcos reflexos espinais.

A ablação do córtex visual de ratos praticados por Goodman \& Horel (1966) ${ }^{11}$ permitiu a estes investigadores verificar a existência de brotamento axônico nas projeçōes do trato óptico; este brotamento era mais evidente $\mathrm{e}$ intenso em direção às áreas de convergência do maior número de fibras do sistema (metade caudal do gânglio geniculado e porção medial do núcleo pré-tectal). Tais observações fizeram com que os autores admitissem a provável existência de correlação entre brotamento axônico e plasticidade funcional.

Segundo Raisman (1969) ${ }^{25}$, a ocorrência de alterações no sistema nervoso central é de suma importância não só para a avaliação do grau de recuperação funcional após lesões destrutivas, como também para a correlação entre as bases anatômicas e os vários métodos de reeducação. Embora o sistema nervoso periférico, sob condições favoráveis, regenere rápida e am- 
plamente (cerca de $2,5 \mathrm{~mm} / 24 \mathrm{~h}$, em média), a regeneração do sistema nervoso central é menos eficaz, tornando-se progressivamente mais difícil se se levar em conta a evolução onto e filogenética, interrompendo-se em níveis animais mais superiores, como o do mamífero adulto. Dois tipos de alterações reconstrutivas são possíveis após a interrupção dos axônios: em primeiro lugar, as fibras seccionadas podem enviar brotamentos novos que atravessam a lesão e reestabelecem suas conexões originais. Embora este seja um processo importante mediante o qual os nervos periféricos respondem às lesões, sua ocorrência e funcionalidade no sistema nervoso central é posta em dúvida; na segunda eventualidade ocorre germinação ou crescimento de terminais de fibras adjacentes para as zonas desaferentadas (descrito em vários locais do sistema nervoso periférico e sistema nervoso central), sendo que neste tipo é que pode ocorrer a reconstrução e consequente reorganizaçāo anatômica. Em suas investigações, Raisman observou ainda que as fibras seccionadas fazem somente uma tentativa abortada para cruzar a lesão; entretanto, heterotopicamente, ocorre reinervação resultante da formação de novos contactos através de fibras intactas e persistentes da zona desaferentada. Este tipo de reorganização fez com que surgissem dúvidas a propósito da especificidade entre as conexões sinápticas.

Se a reinervação ocorrer da forma acima descrita haverá, em última análise, perda de especificidade entre as novas conexões, pois estas não conseguem desempenhar as mesmas funçōes antes cumpridas por determinadas fibras específicas; é bem possivel, entretanto, que, diante de uma população heterogênea de terminações de fibras nervosas recém-formadas neste local, a zorıa desaferentada venha a readquirir certo grau de seletividade.

$\mathrm{Na}$ tentativa de encontrar o substrato anatômico responsável pela reorganização espontânea, várias pesquisas foram feitas visando a comprovar a existência de regeneração no sistema nervoso central. Para Windle (1962) ${ }^{30}$, estas tentativas foram coroadas de pleno êxito, pois nas secções transversas da medula espinal de ratos, gatos, cães e macacos, ficou comprovada a existência de brotamento de axônios que atravessam a cicatriz resultante da lesão. No gato, em particular, Windle demonstrou que as fibras regeneradas que atravessam a cicatriz conservam a capacidade de conduzir o influxo nervoso. A generação no sistema nervoso central de animais recém-nascidos se faz a partir de neuroblastos ainda indiferenciados no momento da lesão (mecanismo raro); na lesão da medula espinal, Liu \& Chambers (1958) ${ }^{23}$ observaram brotamento provindo de axônios adjacentes e intactos (regeneração colateral); em mamifero adulto a regeneração é término-terminal e se faz a partir dos próprios axônios lesados, cuja potencialidade individual é desconhecida. Thulin (cit. por Guth \& Windle ${ }^{12}$ ) demonstrou que axônios de neurônios propriospinais brotam e atravessam o segmento medular correspondente ao nível da lesão para fazer sinapse com outros neurônios propriospinais.

$\mathrm{Na}$ última década os progressos e esforços em várias áreas do conhecimento permitiram esclarecer alguns aspectos ainda obscuros a propósito da regeneração no sistema nervoso. Segundo o princípio de Weiss (cit. por 
Guth \& Windle ${ }^{12}$ ) a regeneração é manifestação do crescimento permanente de neurônios; este principio neurobiológico resultou de estudos a propósito de fluxo axoplasmático mediante o emprego de substâncias radioativas. Se o cilindreixo é seccionado, o segmento distal degenera, enquanto há cresci. mento permanente e regeneração a partir da porção proximal. Desde que a regeneração é mera manifestação de fluxo axoplasmático que ocorre em neurônios periféricos, é lógico e normal admitir-se sua ocorrência também nos processos de regeneração do sistema nervoso central; além disso, e ao contrário do que antes se supunha, os axônios regenerados fazem conexões sinápticas menos rígidas; a despeito da regeneração de axônios no sistema nervoso central, bem como de suas reconexões sinápticas, a recuperação funcional é ainda bastante limitada por fatores até agora desconhecidos.

Segundo Gerard (1972) ${ }^{8}$, são necessários estudos exaustivos visando a tornar a "terapêutica da regeneração" uma realidade. Dentre os vários aspectos destaca: a) o potencial de regeneração pode ser parcialmente dependente da idade, pois o sistema nervoso imaturo possui maior grau de plasticidade; b) a importância de células não neuronais, isto é, de suporte do sistema nervoso central para o processo de regeneração; c) questões relacionadas com a especificidade de conexões, pois não se sabe ainda como se comportam as fibras regeneradas, isto é, se a fibra é específica para a unidade com a qual se conecta, ou vice-versa ou, então, se cada fibra é permanentemente programada para uma dada conexão em particular ou como as células se situam umas em relação às outras.

Admitindo que a regeneração constitui o mecanismo básico ideal para explicar o retorno de funçōes após lesão do sistema nervoso central, outras alternativas devem ser consideradas para torná-la uma realidade. O sistema nervoso central sofre modificações com o decorrer do tempo para melhor utilizar os circuitos locais necessários para a função. A compreensão dos parâmetros fisiológicos de tal situação poderá abrir novos horizontes nas possibilidades terapêuticas para a utilização destes circuitos reorganizados.

\section{FUNÇAO VICARIANTE}

A teoria da "função vicariante", proposta por Fritsch \& Hitzig em 1870 (cit. por Lashley ${ }^{20}$ ) pode ser considerada pioneira dentre as várias tentativas feitas visando a explicar os mecanismos básicos responsáveis pela recuperação de funções após comprometimento do sistema nervoso central. Segundo essa teoria, "ligações indiretas entre os vários centros encefálicos" estariam envolvidas no intrincado mecanismo responsável pelo retorno espontâneo de funções. Fritsch \& Hitzig, após ablação do córtex motor de um dos hemisférios cerebrais de cães, observaram que os membros do hemicorpo contralateral ficavam totalmente paralisados; decorrido algum tempo a motricidade retornava e o animal recuperava em grande parte a capacidde motora perdida. A hipótese aventada pelos referidos autores era de que o retorno da função motora decorria da vicariância desenvolvida pelo 
córtex motor do hemisfério oposto. Essa teoria conexionista ou de associação entre os vários centros cerebrais desempenhou papel importante nas tentativas subsequentes que visavam a explicar os mecanismos de retorno espontâneo de funções. Jackson ${ }^{15}$ já havia previsto, em 1874, que há relação entre recuperação e massa residual do sistema nervoso.

A teoria da função vicariante deixou o terreno filosófico e passou a ter bases científicas quando Jacobsen (1931) 16 investigou as influências das áreas frontal e pré-frontal sobre o aprendizado e a retenção de hábitos manipulatórios e discriminativos. Jacobsen submeteu macacos a um mesmo tipo de treinamento e verificou que a retenção dos vários tipos de hábitos discriminativos não sofria qualquer tipo de alteração após ablação das áreas frontal e pré-frontal do córtex cerebral. Diante de tais fatos passou a admitir que, em sistema íntegro e previamente subordinado às áreas lesadas, desenvolvia-se de novo a capacidade para funções mediatas.

Lashley (1938) ${ }^{20}$ submeteu os membros de um hemicorpo de um macaco a treinamento intensivo e durante todo o período em que durou a experiência os membros do hemicorpo contralateral foram imobilizados. A seguir lesou o hemisfério contralateral, promovendo a paralisia dos membros submetidos a treinamento. No pós-operatório imediato observou a transferência dos tipos de movimentos aprendidos para os membros que haviam sido imobilizados durante o período de experimentação. Diante desses fatos, Lashley passou a admitir que a recuperação da função se deve à existência de arcos reflexos múltiplos e com funções semelhantes, responsáveis pela equipotencialidade das áreas de associação do córtex cerebral.

Bucy (1934) ${ }^{4}$, mediante ablação de extensas áreas do córtex motor, demonstrou que é possivel promover a perda total e irreparável da função motora, embora acreditasse que, dentro do sistema, poderia ocorrer a "substituição funcional" em decorrência da interrelação de estruturas córtico-subcorticais. Glees \& Cole (1950) ${ }^{9}$ fizeram lesões circunscritas e seletivas no córtex motor (área 4) e, diante dos resultados, afirmaram que o córtex não funciona como um mosaico, mas sim, em se tratando de movimentos, tem tendência a atuar de maneira mais primitiva e menos diferenciada, o que é possivel em decorrência das conexões plurissegmentares entre a área 4 e os niveis mais inferiores, particularmente os segmentos medulares.

Rexed (1952) ${ }^{27}$ determinou detalhadamente os locais de terminação espinal das fibras descendentes nas camadas ou lâminas citoarquitetonicamente distintas da substância cinzenta da medula espinal. Brodal \& Pompeiano (cit. por Brodal ${ }^{2}$ ), em estudos experimentais realizados em gatos nos quais promoviam lesões corticais, determinaram a origem e terminação das fibras corticospinais, corticorrubras e rubrospinais. Rinvik \& Walberg (cit. por Brodal ${ }^{2}$ ) demonstraram que as projeções rubrospinais, bem como as corticorrubras, são somatotopicamente organizadas, chegando à importante conclusão de que os locais onde terminam as fibras rubrospinais coincidem com aqueles onde terminam as fibras corticospinais (provindas do córtex motor), fato esse também de suma importância por que as fibras corticorrubricas têm origem na mesma região cortical em que se inicia a via corticospinal. Além 
disso, tanto as fibras corticospinais como as rubrospinais, ao atingirem a medula espinal (no gato), fazem sinapse com todos os tipos de células que constituem as lâminas V, VI e VII de Rexed.

A demonstração, por Walberg \& Brodal (1953) ${ }^{28}$, Chambers \& Liu (1957) ${ }^{6}$ e Kuypers (1958) ${ }^{18}$, de que os tratos corticospinal e corticorrubrospinal têm origem e terminaçāo semelhante sugere uma pergunta óbvia: até que ponto a segunda via intervém na execução de movimentos voluntários ágeis e delicados? A resposta à pergunta cria mais dúvidas que esclarecimentos, visto que o trato corticorruborspinal no homem é rudimentar e, enquanto no gato não existem terminações diretas do trato corticospinal em mctoneurônios do corno anterior (sendo as conexões feitas por interneurônios), em macacos algumas conexões se fazem diretamente com os motoneurônios, permitindo que eles sejam atingidos de imediato por influxos corticospinais.

A possibilidade de "função vicariante" foi revista por Lawrence \& Kuypers (1968) ${ }^{21,} 22$ e Brodal (1973) ${ }^{3}$ sendo, entretanto, baseada em semelhanças anatônicas observadas em zonas terminais de diferentes tratos descendentes, não encontrando equivalência em relação ao comportamento dos movimentos. Lawrence \& Kuypers estudaram os efeitos de lesões bilaterais do feixe piramidal sobre a motricidade de macacos com liberdade de movimentos, verificando que o encéfalo controla os movimentos mediante "vias principais" córtico-subcorticais. No pós-operatório imediato os animais conseguiam sentar-se e manter a cabeça ereta, ficar em pé, andar, elevar-se em barras horizontais e subir nas grades; não conseguiam, porém, usar as extremidades, particularmente as mãos, embora conseguissem usá-las para agarrar e para trepar nas grades da jaula; três semanas após a operação retornava a capacidade para utilizar livremente as extremidades e os animais conseguiam alcançar com precisão e com ambas as mãos, pedaços de alimentos mediante o fechamento harmônico de todos os dedos da mão utilizada. A princípio a preensão fazia parte do movimento, que tinha carater global e consistia em uma circundação em forma de gancho de todo o membro; iniciada essa "recuperação suplementar" a preensão tornava-se progressivamente independente do movimento global do membro; finalmente, os macacos conseguiam fazer a extensão completa de cada um dos membros, com o punho em ligeira dorsiflexão e os dedos semifletidos e abduzidos; nesta posição estável conseguiam abrir e fechar as mãos no ato de apanhar pedaços de alimentos. Entretanto, mesmo após período longo de recuperação que durava onze meses ou mais, nunca conseguiam recuperar os movimentos individuais dos dedos; além disso, todos os movimentos eram mais lentos e a fadiga precoce, persistindo dificuldade para extensão dos dedos e, portanto, para liberar o alimento apreendido, embora no ato de subirem ou se elevarem conseguissem, aparentemente, liberar-se com relativa facilidade. A sequência de retorno da função dos membros comprometidos após a lesão unilateral do trato piramidal era em tudo semelhante à dos macacos submetidos a lesão bilateral. Tais dados sugerem a possibilidade de que, após a interrupção uni ou bilateral do trato piramidal, há retorno de vários movimentos e que, na ausência desse trato, o controle dos movimentos seria devido (em substituição) a circuitos do tronco 
cerebral que atuam sobre a medula espinal. Por outro lado, a persistência de deficits, tais como lentidão e perda da capacidade de mover individualmente cada dedo, indicaria que as vias corticospinais sāo essenciais às características normais de velocidade e agilidade dos movimentos e, principalmente, do elevado grau de fracionamento dos movimentos dos dedos e da mão. Lawrence \& Kuypers concluem que, após lesão dos tratos piramidais, embora ocorra retorno de atos motores individuais do membro superior, jamais há retorno da capacidade de mover cada um dos dedos de per si. Isso pode indicar que as principais vias descendentes são representadas pelas fibras corticospinais que terminam diretamente nos motoneurônios, particularmente naqueles que inervam a musculatura distal. Por outro lado, as fibras descendentes do tronco (núcleos laterais e núcleo rubro) terminam na zona internuncial da medula espinal. Assim, no gato, no qual não existem fibras corticospinais diretas, não se observam movimentos em separado de cada dedo. O conceito de que as conexĉes corticomotoneuronais diretas constituem o substrato dos movimentos de cada dedo foi reforçado pelos mesmos Lawrence \& Kuypers (1968) ${ }^{22}$, mediante a verificação de que, em macacos nos quais persistiam algumas fibras piramidais, ocorria retorno parcial da movimentação digital, recuperação mais rápida dos movimentos do membro correspondente e, surpreendentemente, maior velocidade e agilidade de movimentação.

Em estudos experimentais mais recentes (1972) realizados em macacos, Goldberger ${ }^{10}$ procura explicar os mecanismos responsáveis pela recuperação de movimentos após lesões do sistema nervoso central. Para tanto observou o comportamento do reflexo de preensão patológico (grasping reflex ou reflexo de preensão forçada) que, de forma semelhante a outros sinais neurológicos, tende a desaparecer nos casos em que a evoluçāo se faz no sentido da recuperação. Tal recuperação parece refletir a equipotencialidade das áreas motora e pré-motora no controle da motricidade. Esta explicaçāo é até certo ponto contraditória quando comparada com os dados fornecidos pelas observações de clínica pois, segundo estes dados, a preensão forçada resulta de lesões da área 6 (pré-motora) e desaperece quando o processo lesional se expande e atinge a área 4 (motora).

Em se tratando de comprometimento de movimentos e tomando por base as investigações de Chambers \& Kozart $^{7}$ e de Beck \& Chambers $^{1}$ para se caracterizar de forma precisa a natureza de um dado deficit a par da utilização de exames neurológicos é necessário o emprego de técnicas de condicionamento que permitam investigar as alterações do comportamento motor. Visando a investigar estas alterações, Goldberger fez avaliações não só de reflexos como também de movimentos condicionados e obesrvou as alterações no comportamento motor (particularmente da preensão forçada), bem como das influências que as várias forções do sistema nervoso exercem durante o retorno da função motora. Mediante este critério procurou analisar as discrepâncias de interpretação entre a preensão forçada de animais de laboratório e aquela observada em clínica; além disso, propõe outra teoria para explicar a recuperação espontânea da função que, segundo ele, não se deve 
à formação de novas conexões (reorganização espontânea), nem de tratos que assumem a responsabilidade por novas funções (vicariância).

Segundo a teoria proposta por Goldberger ${ }^{10}$, porções integras do sistema nervoso, até então em estado latente, podem ser seletivamente ativadas em resposta à destruição de outras áreas. Investigando em macacos o comportamento do reflexo de preensão em suas diferentes fases evolutivas após lesões corticais associadas à piramidotomia, o autor procurou identificar as alteraçōes que os movimentos sofriam durante a recuperação espontânea. Os mecanismos fisiológicos desenvolvidos e reconhecidos como responsáveis pelos resultados finais que são semelhantes entre si, isto é, exagero do reflexo e do comportamento motor, permite admitir que a função da área de tecido destruída era inibidora. Se, entretanto, a anormalidade persistir ou se desaparecer durante a evolução (retorno da função inibidora), algo ocorreu, isto é, houve alguma alteração funcional que é responsável pela recuperação comportamental.

Os resultados observados por Goldberger ${ }^{10}$ visando a explicar o retorno da funçũo da mũo (Farticularmente na preensão normal) podem ser assim resumidos: a) o trato piramidal inibe a preensão e os reflexos de estiramento miotáticos sendo que, durante a fase de recuperação, a persistência da hiperatividade dos reflexos dos 4 últimos dedos e do polegar, bem como da "função (inibidora) vicariante" através da pirâmide são discordantes; b) dados pouco convincentes não permitem acreditar que haja reorganização funcional, isto é, regeneração por brotamento colateral, pois sua ocorrência implicaria em modificação completa da sindrome piramidal; c) os sinais evidenciados nas lesões piramidais acrescidos aos da área 6 são sob todos os aspectos semelhantes àqueles de lesões piramidais puras; $d$ ) embora a recuperação de um dado tipo de função motora seja medida pelo piramidal, ela não requer necessariamente que o trato piramidal desenvolva todas as suas propriedades funcionais, dentre os quais, por exemplo, a inibição da preensão; e) o elemento básico da recuperação da inibição encontra-se comprometido no reflexo de preensão e é representado pela insistência em usar o reflexo antagonista, ou seja, a evitação táctil; f) quando o animal com lesão na área 6 consegue ultrapassar a fase I (táctil) da preensão patológica mediante exagero da evitação táctil, torna-se possivel ultrapassar a fase II (proprioceptiva); g) a função perdida (inibição reflexa) após lesão da área 6, perdura durante a recuperação e o retorno da função decifitária, isto é, a liberação da preensão normal poderá ser obtida mediante mecanismos fisiológicos que não sejam subordinados à evitação táctil reflexa; $h$ ) tal hipótese requer que diferentes mecanismos fisiológicos sirvam a uma função final comum sem que haja necessidade de fixar determinadas funções para um dado trato do qual habitualmente não se utilizam.

Dados sugestivos que se somam aos anteriores foram observados em macacos cuja lesão inicial foi praticada na região pós-central; no pós-operatório imediato e durante cerca de duas semanas a evitação não foi obtida; entretanto, se a lesão inicial fosse praticada na área 6 e a recuperação prosse- 
guisse, a partir do momento em que a área pós-central era lesada, no pós-operatório imediato a preensão forçada reaparecia e perdurava uma semana; por outro lado, as lesões ventrolaterais da medula espinal (zonas envolvidas na exacerbação da evitação táctil) promoviam de imediato o desaparecimento (inibição) de todos os vestígios de preensão forçada naqueles animais que, após lesão da área 6, apresentavam recuperação parcial dos efeitos desta lesão; o sistema nervoso pode recuperar-se de maneira diferente, na dependência da anátomo-fisiologia das áreas destruídas e das regiões corticais remanescentes; os mecanismos mediante os quais a evitação táctil pode ser obtida e seletivamente desenvolvida ainda são desconhecidas.

Segundo Brodal (1973) ${ }^{3}$, é espantosa a especificidade da organização morfológica do sistema nervoso central. Quanto mais conhecida se torna a estrutura do cérebro, tanto mais nos convencemos de quão esta especificidade pode ser levada a níveis cada vez mais minuciosos. E regra e não exceção que, no sistema nervoso central, por menor que seja um núcleo ele pode ser subdividido em porçōes que diferem quanto à citoarquitetura, arquitetura glial, vasoarquitetura, conexões de fibras, disposição sináptica e atividade bioquímica, constituindo-se em complemento morfológico para a localização da função no sistema nervoso central. Assim, pequenas zonas em regiōes neoformadas podem apresentar diferenças em suas funções, bem como em sua estrutura, podendo-se considerar, como exemplos, as áreas 17, 18 e 19 do córtex visual com suas várias camadas, e a medula espinal. O sistema nervoso pode ser considerado, portanto, como sendo constituído por grande variedade de unidades menores, cada uma das quais com sua organização morfológica particular e sua função específica.

Se nossa atenção for dirigida para as conexões de fibras do sistema nervoso central estaremos diante de outro aspecto de sua organização: a multiplicidade sináptica. De regra cada pequena região recebe fibras de várias outras e, de forma semelhante, envia fibras que se dirigem para várias regiões. Existem, sem dúvida, enormes diferenças quantitativas entre os vários contingentes de fibras aferentes e eferentes de um núcleo; entretanto, a análise das conexões de fibras do sistema nervoso central como um todo permite supor que existem condições do ponto de vista morfológico para que um impulso de uma dada porção do cérebro possa ser transmitido ao longo de rotas circunvizinhas de complexidade variável para qualquer outra parte do sistema nervoso central. As interconexões das mais variadas estruturas permitem, provavelmente, cooperação e integração de funções entre si, tornando até certo ponto injustificável descrever uma dada porção do cérebro como sendo simplesmente "motora" ou "visual". Para citar um exemplo, os colículos superiores são só recebem um contingente maior de fibras ópticas, mas também atuam sobre o córtex acústico, o córtex motor, o córtex somatosensitivo e de associação, a medula espinal, a formação reticular e outros locais.

Se de um lado a maioria de pequenas unidades do sistema nervoso central podem ser identificadas como um todo ao se considerar sua função e estrutura particular, por outro lado elas são amplamente interconectadas e 
funcionalmente dependentes umas das outras. Tais dados permitem supor que a destruição de uma porção do cérebro resulta em alterações de várias funções além daquelas inerentes à região especificamente lesada.

Em clínica neurológica tal ponto de vista trouxe como consequências: a) em qualquer lesão cerebral ocorrem distúrbios de função que, segundo os pontos de vista correntes, não são necessariamente servidas pela porção lesada; b) estas alterações funcionais secundárias ou indiretas podem ser pequenas e muitas vezes escapam ao reconhecimento pelo exame neurológico de rotina, mas estudos sistemáticos de tais alterações mediante métodos adequados podem fornecer dados valiosos a propósito dos mecanismos das funções do sistema nervoso central e fornecem conceitos suplementares adquiridos pela experimentação em animais de laboratório.

Desde que a regeneração de axônios centrais em mamíferos superiores nunca foi demonstrada de forma convincente, a hipótese de que fibras integras substituem as lesadas, aparentemente é mais aceitável.

Com base no conceito da grande capacidade de reserva do cérebro pode-se admitir que, após uma lesão cerebral, algumas células nervosas que não funcionavam tornam-se ativas. Se realmente tais células em estado de latência em um cérebro que atingiu seu pleno desenvolvimento podem escapar à atrofia de desuso é outro aspecto importante a considerar. É mais provável que a "substituição" de células lesadas implique em que as intactas e remanescentes continuam em pleno funcionamento e estabelecem novas sinapses nos locais onde as fibras destruídas antes exerciam sua ação. Wall \& Egger (1971) ${ }^{59}$ concordam com esta hipótese e estudos recentes de anatomia demonstram que realmente é o que ocorre.

Raisman (1969) ${ }^{25}$ e Raisman \& Field (1973) ${ }^{26}$, mediante microscopia eletrônica, observaram os efeitos da interrupção de dois contingentes de aferentes para os núcleos septais no rato: se um dos tratos for seccionado as fibras do outro se estendem para as sinapses desnudas do local pertencente àquelas fibras do trato que foi seccionado. Embora ocorra uma reinervação mediante este processo, é claro que existe uma perda considerável na especificidade das conexões neuronais. No homem pode-se supor que ela se estenda por um período muito longo. Estas hipóteses a respeito de regeneração parecem aplicáveis à melhora da hemiparesia após acidente cerebral, especialmente em fases tardias da evolução, quando desapareceu o bloqueio inicial funcional e temporário de algumas fibras corticífugas lesadas pelo edema. Segundo a hipótese de Raisman, parece muito provável que parte da recuperação seja devida ao fato de que a terminação sináptica do trato corticospinal preservado, e provavelmente de outras fibras do sistema nervoso central, formam novos terminais que substituem e ocupam as zonas sinápticas desnudas dos motoneurônios. Paulatinamente, maior número de motoneurônios originalmente desprovidos de inervação cortical irão sendo reinervados. Processo correspondente poderá ocorrer com outros eferentes corticais em partes interrompidas, tais como os tratos corticorúbrico, reticular e pontino.

Entretanto, pode-se esperar considerável perda da especificidade; uma fibra reticular pode estabelecer uma nova sinápse com um motoneurônio onde 
existia anteriormente uma fibra pertencente ao corticospinal. Funcionalmente haverá confusão devido à formação de novos tipos de conexões neuronais. Nestas condições, mesmo que o funcionamento do novo mecanismo seja ótimo, nunca poderemos esperar o restabelecimento do comportamento neuronal em seu todo e, portanto, uma recuperação completa da função motora.

Parece provável que o mecanismo supramencionado seja fator importante na recuperação após lesão do sistema nervoso central, tendo aplicação geral válida para o tálamo, córtex e outras regiões. Nada se sabe a respeito dos fatores que estimulam o processo, sendo que os resultados do mecanismo descrito são tanto menos satisfatórios quanto mais complexa for a organização da parte cerebral lesada.

Diante do exposto, pode-se admitir que a ocorrência da regeneração do sistema nervoso central permite que novos circuitos sejam organizados e passem a funcionar de forma semelhante a do centro previamente lesado. Tanto no que concerne à organização funcional como estrutural, a forma é inerente às partes correspondentes, continuando a ser o problema imediato o esclarecimento dos mecanismos e os limites dentro dos quais o fenômeno se processa.

\section{RESUMO}

É feita uma revisão dos mecanismos apontados como responsáveis pela recuperação espontânea da função em pacientes com lesões do sistema nervoso central. São referidas as teorias de reorganização espontânea do tecido nervoso e da função vicariante. E destacada a contribuição experimental das duas últimas décadas, particularmente a dos pesquisadores encabeçados por Windle \& Guth, que demonstraram a possibilidade de regeneração no sistema nervoso central, bem como as de Lawrence \& Kuypers, Brodal \& Goldberger, que advogam a função vicariante como mecanismo provável da recuperação da função.

\section{SUMMARY}

Spontaneous recuperation of function in central nervous system lesions

A rewiev of the mechanims responsible for the spontaneous recuperation of function in patients with lesions of the central nervous sistem is made. The spontaneous reorganization theories of the nervous structures and the vicarious function are also referred to. In the last two decades experimental contributions have been accentuated, specially the one conducted by the group of researchers directed by Windle and Guth, who had shown the possibility of regeneration in the central nervous system, as well Lawrende and Kuypers, Brodal, Goldberger and others, which defended the vicarious function as the probable mechanisms of recuperation. 


\section{REFERENCIAS}

1. BECK, C. H. \& CHAMBERS, W. W. - Speed, accuracy and strenght of forelimb movement after unilateral pyramidotomy in rhesus monkeys. J. comp. physiol. Psychol. 70:1, 1970.

2. BRODAL, A. - Experimental anatomical studies of the cortico-espinal and cortico-rubro-spinal connections in the cat. In Szentágothai — Moderm Trends in Neuromorphology, Symp. Biol. Hung. 5, 1965.

3. BRODAL, A. - Self observations and neuro-anatomical considerations after a stroke. Brain 96:675, 1973.

4. BUCY, P. C. - The relation of the premotor cortex to motor activity. J. nerv. ment. Dis. 79:621, 1934 .

5. BUCY, P. - Neuromechanism controlling skeletal muscular activity and its unsolved problems. Neurosciences Research 1:251, 1968.

6. CHAMBERS, W. W. \& LIU, C. - Cortico-spinal tract of the cat. J. comp. Neurol. 108:23, 1957.

7. CHAMBERS, W. W. \& KOZART, D. - Conditioned tactile discrimination of monkey with corticospinal or pyramidal tract lesions. Anat. Rec. 151:499, 1965.

8. GERARD, R. - Regenerative phenomena in the central nervous system: a symposium summary. J. Neurosurg. 37:129, 1972.

9. GLEES, P. \& COLE, J. - Recovery of skilled motor functions after small repeated lesions of motor cortex in macaque. J. Neurophysiol. 13:137, 1950.

10. GOLDBERGER, M. E. - Restitucion of function in the CNS: the pathologic grasp in macaca mulatta. Brain 15:79, 1972.

11. GOODMAN, D. C. \& HOREL, J. A. - Sprouting of optic tract projection in the brain stem of the rat. J. comp. Neurol. 127:71, 1966.

12. GUTH, L. \& WINDLE, W. F. - The enigma of central nervous regeneration. Exp. Neurol. 37:1, 1972.

13. GUTH, L. \& WINDLE, W. F. - Physiological, molecular, and genetic aspects of central nervous system regeneration. Exp. Neurol. 39:3, 1973.

14. HAYMAKER, W. - Bing's Local Diagnosis in Neurological Diseases. C. V. Mosby Co., St. Louis, 1956, p. 256-258.

15. JACKSON, J. H. - Remarks on the diagnosis and treatment of diseases of the brain. In J. Taylor's "Selected Writings of John H. Jackson". Hodder e Stoughton, Londres, 1932, p. 384.

16. JACOBSEN, C. F. - A study of cerebral function in learning: the frontal lobes. J. comp. Neurol. 52:271, 1931.

17. KENNARD, M. A. - Reorganization of motor function in the cerebral cortex of monkeys deprived of motor and promotor areas in infancy. $J$. Neurophysiol. 1:477, 1938 .

18. KUYPERS, H. G. J. M. - Corticobular connexions to the pons and lower brain stem in man. Brain 81:364, 1958.

19. KUYPERS, H. G. J. M. \& BRINKMAN, J. - Precentral projections to different parts of the spinal intermediate zone in rhesus monkey. Brain Res. 24:29, 1970.

20. LASHLEY, K. S. - Factors limiting recovery after central nervous lesions. J. nerv. ment. Dis. 88:733, 1938.

21. LAWRENCE, D. G. \& KUYPERS, H. G. J. M. - The functional organization of the motor system in the monkey: the effects of bilateral pyramidal lesions. Brain 91:1, 1968.

22. LAWRENCE, D. G. \& KUYPERS, H. G. J. M. -- The functional organization of the motor system in the monkey: the effects of lesions of the descending brain-stem pathways. Brain 91:15, 1968.

23. LIU, C. \& CHAMBERS, W. W. - Intraspinal sprouting of dorsal root axons. Arch. Neurol. Psychiat. (Chicago) 79:46, 1958.

24. McCOUCH, G. P.; AUSTIN, G.; LIU, C. N. \& LIU, C. Y. - Sprouting as a cause of spasticity. J. Neurophysiol. 21:205, 1958.

25. RAISMANN, G. - Neuronal plasticity in the septal nuclei of the adult rat. Brain Res. 14:25, 1969. 
26. RAISMANN, G. \& FIELD, P. M. - A quantitative investigation of the development of collateral reinnervation after partial deafferentation of the septal nuclei. Brain Res. 50:241, 1973.

27. REXED, B. - The cytoarchitectonic organization of the spinal cord in the cat. J. comp. Neurol. 96:415, 1952.

28. WALBERG, F. \& BRODAL, A. - Pyramidal tract fibres from temporal and occipital lobes: an experimental study in the cat. Brain 76:491, 1953.

29. WALL, P. D. \& EGGER, M. D. - Formation of new connexions in adult rat brains after partial deafferentation. Nature (London) 232:542, 1971.

30. WINDLE, W. F. - Regeneration in the central nervous systems. In French \& Porter - Basic Research in Paraplegia. Charles C. Thomas Publ., Springfield (Illinois), 1962, p. 3-8.

Clinica Neurológica - Faculdade de Medicina, Universidade de São Paulo Caixa Postal 3461 - 01000 São Paulo, SP - Brasil. 